\title{
Perceptions des Apprenants par Rapport au Redoublement: Facteurs de l'Échec et du Décrochage d'un Cours
}

\author{
Perceptions of students in relation to repetition: \\ Factors of failure and abandonment of a course
}

\begin{abstract}
Percepciones de los estudiantes en relación con la repitencia: Factores de fracaso y abandono de un curso
\end{abstract}

\author{
Anyela Mariely Gelvez Rivero \\ Étudiante en Langues Étrangères (Anglais-Français) \\ Université de Pamplona \\ Yisel Carolina Vanegas Negrete \\ Étudiante en Langues Étrangères (Anglais-Français) \\ Université de Pamplona
}

\section{Résumé}

Le redoublement et le décrochage sont des phénomènes affectant certains étudiants de la Licence en Langues Étrangères de l'Université de Pamplona en Colombie. Pour comprendre les perceptions des étudiants, cette recherche a adopté une étude phénoménologique et une approche mixte car la combinaison de méthodes quantitatives et qualitatives fournit une meilleure compréhension de l'objet d'étude. Ce projet répond à deux questions principales : quelles sont les perceptions des apprenants de FLE niveau B1 par rapport au redoublement? Quels sont les facteurs causant l'échec et le décrochage d'un cours? Identifiant si les étudiants perçoivent le redoublement comme un facteur positif ou négatif dans l'apprentissage d'une langue étrangère. Nous employons un échantillon composé par 16 participants, âgés entre 18-24 ans. Pour la collecte des données, nous utilisons les instruments suivants : l'enquête, le questionnaire, le groupe focalisé et l'entretien. Pour le processus d'analyse qualitative des données, nous conduisons une analyse phénoménologique, centrée sur les expériences des participants. Nous employons le logiciel MAXQDA afin d'organiser les données d'une manière détaillée en utilisant des codes et des catégories. Les résultats indiquent que le redoublement provoque des conséquences positives et négatives à cause des problèmes économiques et académiques causant d'autres soucis d'ordre psychologiques chez les étudiants.

Mots clés: perceptions des étudiants, redoublement, échec, décrochage

Recepción: 05-02-19 Aceptación: 26-04-19 


\section{Resumen}

La repitencia y la deserción son fenómenos que afectan a ciertos estudiantes de la Licenciatura en Lenguas Extranjeras de la Universidad de Pamplona en Colombia. Esta investigación adoptó un estudio fenomenológico y utilizó un enfoque mixto, ya que la combinación de métodos cuantitativos y cualitativos proporciona una mejor comprensión del objeto de estudio. Este proyecto responde a dos preguntas principales: ¿cuáles son las percepciones de los estudiantes respecto a la repitencia? y ¿̇cuáles son los factores que causan el fracaso y la deserción de un curso? Identificando si los estudiantes perciben la repitencia como un factor positivo o negativo en el aprendizaje de una lengua extranjera. Nosotros empleamos una muestra de 16 participantes, de 18 y 24 años. La recolección de datos se realiza empleando los siguientes instrumentos: encuestas, cuestionarios, grupos focales y entrevistas. Para el proceso de análisis de datos cualitativos se realiza un análisis fenomenológico, centrado en las experiencias de los participantes. Se emplea el software MAXQDA, ya que este hace posible organizar los datos de una manera detallada mediante el uso de códigos y categorías. Los resultados indican que la repitencia provoca consecuencias positivas y negativas, y es causada por problemas económicos y académicos, generando efectos psicológicos sobre los estudiantes.

Palabras claves: percepciones de los estudiantes, repitencia, fracaso, deserción

\section{Introduction}

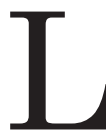

e redoublement est un phénomène qui affecte certains étudiants de la Licence en Langues Étrangères (Anglais-Français) de l'Université de Pamplona en Colombie. Selon Cosnefroy et Rocher (2005) les taux de redoublement au cours de la scolarité obligatoire ont beaucoup diminué depuis 40 ans (p.5). Cependant, les étudiants redoublants présentent des faibles compétences au niveau académique car ils n'acquièrent pas les capacités nécessaires telles que la responsabilité, l'autonomie et la motivation pour apprendre une langue étrangère, c'est pourquoi le redoublement est considéré comme un recours efficace car il représente une seconde chance, pour les apprenants, visant à améliorer leur processus d'apprentissage (Cosnefroy et Rocher, 2005).
Du côté scolaire, le CNESCO (2016) estime qu'en 2013, un élève sur quatre en fin de collège a redoublé au moins une fois, alors qu'en 2004 c'était plus d'un élève sur trois. Cette problématique a généré un taux inégal d'étudiants redoublants en tenant compte leur origine sociale et le contexte économique des familles, comme si la pauvreté était l'une des causes pour les carences cognitives. En plus, le processus de redoublement des étudiants est évidemment complexe car il met potentiellement en jeu des aspects aussi divers que le contexte scolaire, les recommandations politiques, les stratégies des familles, entre autres (Crahay, 1996, 2000 ; Paul, 1996 in Cosnefroy et Rocher, 2005, p.10).

De plus, Paul (n.d) se demande si le redoublement est à tous les coups un moyen efficace de remettre à niveau les étudiants un peu à la traîne et il 
constate que " les apprenants même faibles progressent plus vite quand ils ne répètent pas, surtout en primaire. Par contre, les redoublants profitent de quelque temps d'un effet dopage puis se lassent très vite de revoir des programmes qu'ils ont déjà vu ".

En Colombie, l'UNICEF (2015) focalise son attention sur l'éducation de base et il estime qu'entre le début des années 90 et 2000, le nombre moyen d'années de scolarité achevées est passé de 3 à 3,7 pour les filles et de 3,1 à 3,8 pour les garçons. Chez les femmes, les taux de redoublement et d'abandon scolaire sont inférieurs à ceux des hommes. En milieu urbain, 87\% environ des enfants recevant une éducation primaire passent dans la classe supérieure, contre $78 \%$ en milieu rural.

Du côté des études universitaires, les experts de l'UNICEF (2015) estiment que l'échec a une tendance à provoquer l'abandon des études. Un étudiant qui redouble un semestre au moins deux fois de suite, par exemple, a tendance à abandonner l'université, en raison de sa démotivation, de son insatisfaction et de l'humiliation par rapport aux autres camarades quand ils continuent leur processus apprentissage. Par conséquent, en tant que redoublant, il doit travailler la même thématique à nouveau. Nonobstant, ces experts de l'UNICEF (2015) indiquent aussi que certains étudiants échouent simplement parce qu'ils n'aiment pas l'université et encore moins étudier. De même, s'ils font des efforts, ils ressentent de grandes difficultés à assimiler et à comprendre la matière et les thématiques à apprendre. En revanche, il y a ceux dont les parents n'ont pas les moyens pour payer leurs études. Finalement, les garçons sont les étudiants qui ont une forte tendance à quitter l'université en comparaison avec les filles.

Chauveau et Rogoas-Chauveau (1996) chargés de la recherche CRESAS-INRP, expliquent que l'échec est causé et peut correspondre à six types de problèmes différents présents chez les universitaires. Premièrement, il est lié aux difficultés d'adaptation à la structure scolaire et a une relation avec les perturbations comportementales et relationnelles de certains étudiants. En plus, les conditions socio-affectives compliquées, un deuil, l'anxiété, les soucis d'ordre économique peuvent être à l'origine d'un décrochage scolaire. En deuxième lieu, l'échec est associé aux difficultés d'apprentissage centrées sur les problèmes cognitifs et le manque de compétences provoquant les insuccès dans certains savoirs de base (lire, écrire, calculer) observables dès le cours préparatoire. En troisième lieu, il est dû aux procédures d'élimination ou de relégation sur les orientations négatives, tels que le redoublement et le placement dans une structure ou dans une filière dévalorisée. En quatrième lieu, l'échec est lié aux difficultés de passage d'un cycle à une autre fondées sur le non-accès au lycée puis à l'enseignement supérieur. En cinquième lieu, il est associé à l'insuffisance ou à l'absence de certification scolaire générant qu'un grand nombre d'étudiants ne parvienne pas à obtenir un diplôme d'études secondaires ou d'études professionnelles et sur l'évaluation ou la sanction d'études (examen, diplômes). Enfin, il est centré sur les difficultés d'insertion professionnelle et sociale basées sur la sortie du système scolaire et l'entrée dans le monde du travail. 


\section{Le Cadre théorique et revue de la littérature}

\section{Le Redoublement}

Le redoublement est le fait de recommencer un semestre d'étude, de parcourir une deuxième fois le même programme de son niveau antérieur. Selon Draelants (2006) les faibles compétences académiques des étudiants montrent que le redoublement est un recours pédagogique efficace " comme moyen de traiter les difficultés scolaires au cours de la scolarité obligatoire " (p.9). D'une part, l'auteur analyse la comparaison entre la pensée des personnes face à la pratique du redoublement comme un fait utile et nécessaire au niveau éducatif et sa réalité inefficace concernant le progrès cognitif, la motivation et la méthodologie des enseignants. D'autre part, Cosnefroy et Rocher (2005) indiquent que la plupart des étudiants considèrent le redoublement comme une seconde chance. Cependant, il affecte leur motivation, leurs comportements et leurs stratégies d'apprentissage car une personne - qui tient à redoubler - est incapable de réussir au niveau suivant par manque de connaissances.

D'après le travail de Tejada (2015) il existe trois types d'hypothèses liées au redoublement. La première indique qu'il n'est pas un problème seulement des étudiants car il est relatif à une mauvaise influence de l'entourage ou encore par manque d'investissement de leur part. La deuxième implique qu'il a un impact psychologique puisque les efforts attendus des étudiants ayant une seconde chance ne sont pas suffisants car ils ne sont pas motivés pour améliorer. Dans la dernière hypothèse les étudiants n'associent que ce phénomène à un échec.

\section{L'échec}

L'échec est le processus par lequel l'individu cesse de répondre aux exigences universitaires d'apprentissage et de comportement. Dans le cas français, l'analyse faite des travaux menés par le CEREQ et l'Observatoire de la Vie Etudiante montre que 90000 jeunes abandonnent l'université sans obtenir un diplôme, soit $20 \%$ des inscrits en 2004 (Beaupère, Boudesseul et Macaire, 2009 in Sarfati, 2015). De plus, Beaud (2005) indique que le taux de réussite en premier cycle est imparfait car il omet une donnée essentielle à la compréhension de la problématique de l'échec, celle du taux d'abandon en cours d'année et celle des " étudiants fantômes " qui s'inscrivent formellement à l'université et ne suivent pas les cours. En plus, il identifie trois facteurs susceptibles à contribuer à l'échec : la spécialisation du premier cycle, l'organisation pédagogique et le sous-encadrement des étudiants.

En outre, Sarfati (2015) décrit les trois types de parcours suivis par les étudiants universitaires et qui provoquent l'échec : ceux changeant de filière rapidement, ceux qui choisissent l'université à la carte, et ceux qui cumulent des difficultés. Les apprenants associés au premier parcours se sont inscrits et se tournent vers une filière dans laquelle ils aspiraient aller, tandis que les autres ont des difficultés dans leur processus de sélection universitaire. Finalement, les jeunes qui cumulent des difficultés scolaires et sociales sont confrontés à une violence sociale et symbolique forte. 
D'abord, parce qu'ils se rendent compte que leurs bagages ne leur permettent pas de suivre les enseignements théoriques et abstraits propres aux disciplines académiques, et même le vocabulaire ou les expressions propres aux contenus pédagogiques. Par contre, les étudiants situés dans ce parcours manifestent avec plus ou moins de tact qu'ils ne parlent pas la même langue que les enseignants et ne sont pas au niveau.

\section{Le décrochage d'un cours}

Le décrochage est le fait de quitter l'université sans diplôme ou sans finir les études et c'est un processus cumulatif où le milieu socio familial et fonctionnement/vécu scolaire interviennent. Les caractéristiques individuelles des jeunes influençant leur processus d'apprentissage sont les problèmes de motivation et de discipline voire de comportement montrant des faibles résultats, en plus de l'absentéisme. Ceux-ci les désengagent de l'université, tant au niveau des apprentissages qu'au niveau social (Chenu et Blondin, n.d).

D'ailleurs, les garçons sont - le plus souvent - touchés que les filles par le décrochage (15,3\% contre 11,6 $\%$ au niveau européen en 2014). Dans la situation en Fédération Wallonie Bruxelles, Lafontaine et Crahay (2004) affirment que le décrochage est le dernier maillon de la chaîne, l'ultime réponse que l'étudiant apporte lorsqu'aucune autre " porte de sortie " ne s'ouvre au terme d'un long parcours d'échecs où se succèdent d'abord les redoublements, ensuite les changements d'orientation ou d'université. De même, Monseur et Crahay (2008) signalent que les jeunes en décrochage proviennent souvent d'établissements regroupant beaucoup d'étudiants défavorisés, ce qui engendre des effets d'agrégation, c'est-à-dire " il y a des effets spécifiques sur les performances scolaires par le regroupement d'étudiants d'une même catégorie sociale ou ethnique au sein d'un même établissement» (p. 35).

Dans son étude, Lozano (2013) montre qu'il existe certains facteurs internes et externes qui forcent l'étudiant à abandonner un programme. Premièrement, les étudiants abandonnant le programme possèdent non seulement des difficultés académiques, socio-économiques et personnelles mais qu'il y a d'autres facteurs comme le manque de motivation et les mauvaises décisions au moment de choisir une filière universitaire, générant l'abandon $\mathrm{du}$ processus d'apprentissage d'une langue étrangère. En deuxième lieu, la difficulté que trouvent les étudiants au moment d'apprendre une nouvelle langue, le manque d'intérêt, les inconvénients économiques, les diverses responsabilités de chaque individu et le manque d'éclaircissement des thèmes par les enseignants.

Par ailleurs, le Conseil des ministres de l'éducation de l'Union européenne (2011) détermine les principaux facteurs conduisant à l'abandon et étudie les caractéristiques de ce phénomène, avec des stratégies globales, mesures adaptées aux groupes présentant un risque accru d'abandon. D'une part, les mesures de prévention visent à améliorer l'offre et la qualité de l'éducation. D'autre part, la mesure d'intervention en Wallonie-Bruxelles mise en place vise à transformer les universités en communautés d'apprentissage, en employant 
des systèmes d'alerte rapide, notamment en lien avec l'absentéisme.

Dans le cas colombien, grâce à la mise en œuvre du Sistema de Prevención y Análisis de la Deserción en las Instituciones de Educación Superior-SPADIES, c'est évident que le décrochage est considéré comme un problème d'efficacité et d'équité. SPADIES présente aussi les principaux facteurs affectant la prévalence de ce problème. Ils résident dans les lacunes académiques des étudiants au niveau supérieur car il y a un nombre important de jeunes qui débutent l'éducation supérieur et ne parviennent pas à la compléter. Un autre facteur est lié aux difficultés économiques où la majorité de l'argent investi pour aider les étudiants dans des programmes non complémentaires est gaspillé. Le dernier facteur est associé au manque d'orientation professionnelle, un processus qui faciliterait la sélection d'un programme académique basé sur l'identification des capacités et des motivations; ainsi que d'attitudes, d'aptitudes et d'attentes des étudiants liées à la connaissance de ce qu'ils veulent être, la gestion de leur temps libre et leur projet de vie (Ministerio de Educación Nacional de Colombia, 2015).

\section{Méthodologie}

\section{La Population}

Cette étude s'est développée au sein de la Licence en Langues Étrangères (Anglais-Français) de l'Université de Pamplona engagée dans un développement humain responsable. Les apprenants - faisant partie de ce projet - suivent des études en langues étrangères et en langue espagnole et communication.

Les chercheurs ont utilisé un échantillon par quotas suivant certains nombres de caractères, ils ont travaillé avec 7 étudiants redoublants du troisième semestre, niveau B1 et 9 étudiants décrocheurs, divisés en 12 femmes et 4 hommes, âgés entre 18-24 ans afin de constater toutes les perceptions. Cet échantillon a été construit en respectant la distribution de la population, il a été choisi de façon à constituer une image aussi fidèle que possible de la population totale, en comprenant les implications du redoublement sur l'apprentissage d'une langue étrangère (Hababou n.d).

\section{L'approche}

Dans cette étude les chercheurs ont adopté une méthode mixte donnant l'opportunité d'analyser les perceptions des apprenants par rapport au redoublement et de les quantifier tenant compte les implications personnelles et sur leur vie académique (Johnson et Onwuegbuzie, 2004). D'une part, cette méthode présente un design avec des hypothèses philosophiques guidant l'orientation de la collecte, l'analyse, le mélange de données qualitatives et quantitatives dans une seule étude. Ce qui a favorisé la reconnaissance des émotions, des sentiments ainsi que des comportements et des expériences personnelles des étudiants (Creswell et Plano Clark, 2006, p. 5). D'autre part, les chercheurs ont employé une étude phénoménologique contribuant à comprendre un phénomène à partir des perceptions et des perspectives, et même à analyser les expériences 
des participants et à souligner l'intentionnalité de la conscience où les expériences contiennent à la fois l'apparence extérieure et la conscience intérieure basée sur la mémoire, l'image et le sens du phénomène Creswell (1998, p. 52).

\section{Les instruments}

Les chercheurs ont mis en place quatre instruments pour la collecte des données de ce projet. D'une part, deux instruments qualitatifs, le groupe focalisé ayant un nombre de personnes dans une conversation centrée sur un sujet ou un champ d'intérêt défini (Kitzinberg, Marková et Kalampalikis, 2004). D'après ces auteurs, cet instrument a aidé les chercheurs à analyser les comportements des étudiants redoublants face au sujet développé car il favorise l'expression des perceptions, des attitudes, des croyances, des sentiments, des aspirations, des résistances et des intérêts présents ayant les personnes du groupe. Les échanges favorisent l'émergence de connaissances, d'opinions et d'expériences comme une réaction en chaîne grâce à la réunion de personnalités diverses favorisant l'expression et la discussion d'opinions controversées (Touboul, n.d). L'entretien a été utilisé comme une autre méthode de recueil d'information qualitative appuyant les chercheurs à obtenir des informations sur des faits ou des représentations des étudiants pour vérifier la pertinence, la validité et la fiabilité des données face au phénomène étudié. Elle consiste à des entretiens oraux, individuels ou de groupes, avec plusieurs personnes sélectionnées (Ketele et Roegiers, 2015).

D'autre part, deux instruments quantitatifs tels que, le questionnaire et l'enquête ont été utilisés. Le questionnaire est un instrument centré sur les questions et les témoignages des participants, rendant possible de connaitre une situation et de l'analyser. Dans la phase préliminaire, les chercheurs ont présenté des hypothèses par rapport au sujet traité, ils ont posé donc de questions et les ont développées pendant l'étude pour collecter les données. De la même manière, les participants ont répondu à certaines questions de type ouvertes et fermés, en fournissant donc leurs informations personnelles (Creswell 2012, p.382-383).

L'enquête a été un autre instrument facilitant la collecte des données sur une forme récapitulative utile, qui décrivait les attitudes, les opinions, les comportements, les expériences ou les caractéristiques des apprenants (Statistique Canada, 2003). C'est une activité organisée et méthodique sur des caractéristiques d'intérêt d'une partie ou de la totalité des unités d'une population à l'aide des concepts, des méthodes et des procédures bien définis (Creswell, 2012).

Pendant le processus d'analyse des données, les chercheurs ont fait face à la richesse des données qualitatives; c'est pourquoi ils ont mené une analyse phénoménologique, centrée sur les expériences vécues des participants afin de produire une description et de déterminer la forme et la nature de la réalité de ce phénomène. D'après Giorgi (1997) in St-Germain, Delpêche, et Mercier (2009) les données qualitatives aident les chercheurs à apprécier plutôt les pensées des participants. En outre, il propose au chercheur d'effectuer une réduction phénoménologique qui se reflète tout au long des étapes de l'analyse des données tenant compte 
qu'elle contribue alors à une meilleure précision des résultats en relevant essentiellement la conscience des participants face au phénomène en cause. Cet auteur utilise cinq étapes pour développer le processus de l'analyse phénoménologique des données; à savoir: recueillir les données, faire une lecture minutieuse, diviser les données en unités de signification, présenter l'organisation et l'énonciation des données et synthétiser les méta-thèmes.

\section{Résultats}

\section{Effets psychologiques du redouble- ment chez les apprenants}

Du côté psychologique, le redoublement peut être perçu négativement par certains étudiants car il est " en général ressenti par eux comme une punition, comme un stigmate, qu'ils porteront longtemps et qui ne sera pas sans conséquence sur la suite de leur carrière" (Paul, 1996, p.11). Mais, il y en a d'autres qui le voient comme une mesure positive, car il « s'agit de redonner une chance pour mieux réussir» (p.46). C'est pour cela que nous présentons les effets psychologiques du redoublement chez les apprenants redoublants d'un cours de FLE.

Premièrement, nous constatons que le redoublement d'un cours provoque des effets négatifs chez les étudiants car les participants expliquent qu'ils peuvent se sentir démotivés ou se sentir seuls quand ils refont le cours. Le participant 1 expose que " lorsque vous avez de faibles notes ou si la relation que vous avez avec l'enseignant n'est pas la meilleure, cela vous décourage, alors vous perdez tout intérêt pour le cours et vous ne travaillez pas à la maison, c'est-à-dire, vous ne faites aucun travail». D'ailleurs, le participant 2 exprime que " vous vous sentez seul parce qu'avec les camarades de classe que vous avez commencé, ils sont dans des semestres plus avancés et pour cette raison vous devez faire de nouvelles amitiés ". De plus, les participants $(3,4,5)$ mentionnent que le redoublement affecte leurs comportements car ils ressentent une série d'émotions telles que la frustration, la déception, le désintérêt, la motivation puisqu'ils améliorent leur processus d'apprentissage en comprenant plus clairement les sujets, et aussi tous les aspects émotionnels liés à la perte et à l'échec. En plus, les étudiants affirment qu'en répétant le cours, ils ont la possibilité de clarifier leurs doutes et ainsi obtenir de meilleures notes.

Par ailleurs, le redoublement provoque une conséquence défavorable liée au jugement et à l'isolation chez les étudiants car ils se sentent jugés par les professeurs, les camarades de classe et les membres de leur famille. Les parents ressentent de la tristesse et de la frustration lorsque leurs fils doivent répéter le cours. Le participant 3 exemplifie cette conséquence " dans mon cas, il y avait une fois qu'un enseignant nous laissait de côté pour être redoublants, c'est-à-dire, qu'il expliquait tout le temps aux étudiants qu'il avait accompagné depuis le premier semestre et vous cherchiez d'autres moyens pour étudier et travailler chez vous de manière autonome».

Au début de la licence, les étudiants sont motivés car ils sont pleins de vie, de joie, de rêves, d'espoir; mais pendant le déroulement de leurs études, c'est le contraire, parce qu'ils se sentent 
frustrés de savoir que leurs camarades progressent et ils ne le font pas, et ils sont toujours dans la même situation où ils ont de mauvaises performances. Il existe aussi la peur de rencontrer de nouvelles personnes, les mêmes professeurs ou des enseignants différents, de se sentir jugés ou marqués par ceux qui s'ajustent bien au système.

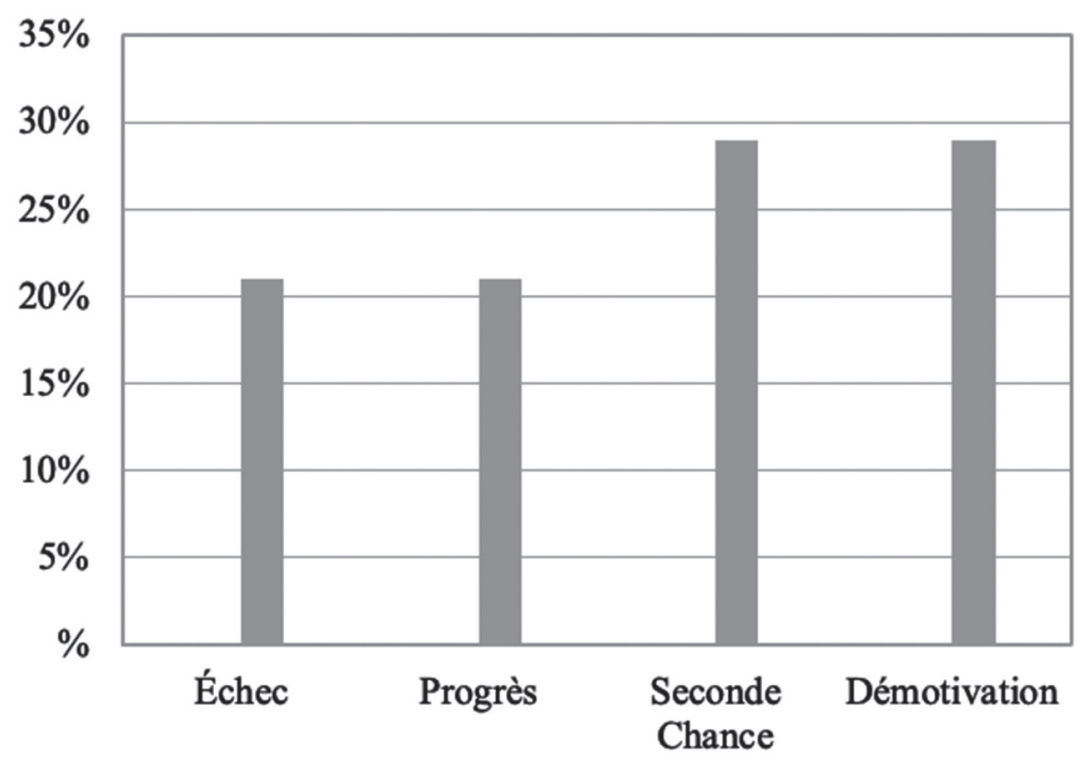

Figure 1. Que représente le redoublement?

En outre, selon les expériences personnelles des participants, le redoublement est vu dans un 50\% comme un facteur positif, où on voit que $29 \%$ est associé à une seconde chance et $21 \%$ indique un progrès. Cependant, il est aussi vu dans un $50 \%$ comme un facteur négatif car $29 \%$ représente la démotivation dans le processus d'apprentissage d'une langue étrangère et $21 \%$ est lié à l'échec (Figure1).

De la même manière, ceci cause aussi une altération dans la confiance et la motivation des étudiants pour continuer le cours. Par exemple, les participants redoublants mentionnent que les facteurs négatifs présents dans leur vie académique, après avoir échoué un cours sont $20 \%$ tristesse, $19 \%$ démotivation, $16 \%$ peur de ne plus réussir et frustration, $13 \%$ stress, $10 \%$ perte de temps et $3 \%$ faible estime de soi et inutile (Figure 2); tandis que pour les décrocheurs expriment que les facteurs négatifs présents après avoir changé de programme sont $37 \%$ perte de temps, $27 \%$ frustration et aucune raison, $9 \%$ peur de ne plus réussir. Cependant, les apprenants n'ont pas été associés à la démotivation, à la tristesse, au stress et à la faible estime de soi (Figure 3). 


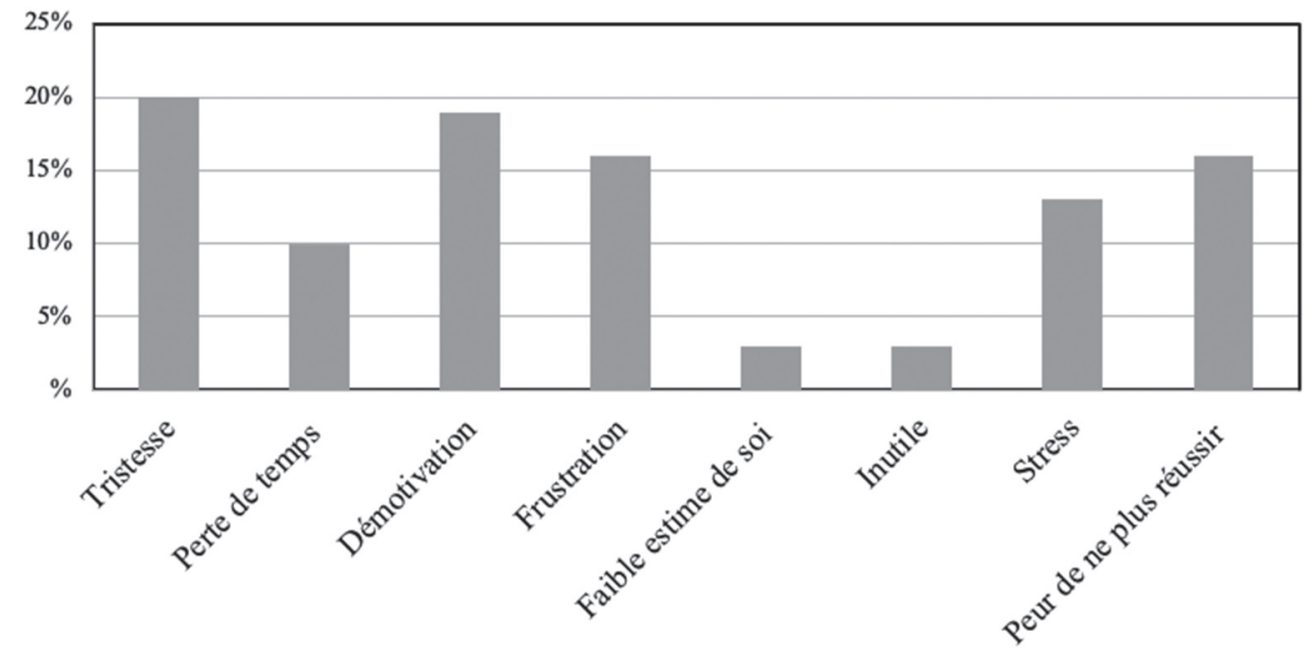

Figure 2. Facteurs négatifs présents après avoir échoué un cours.

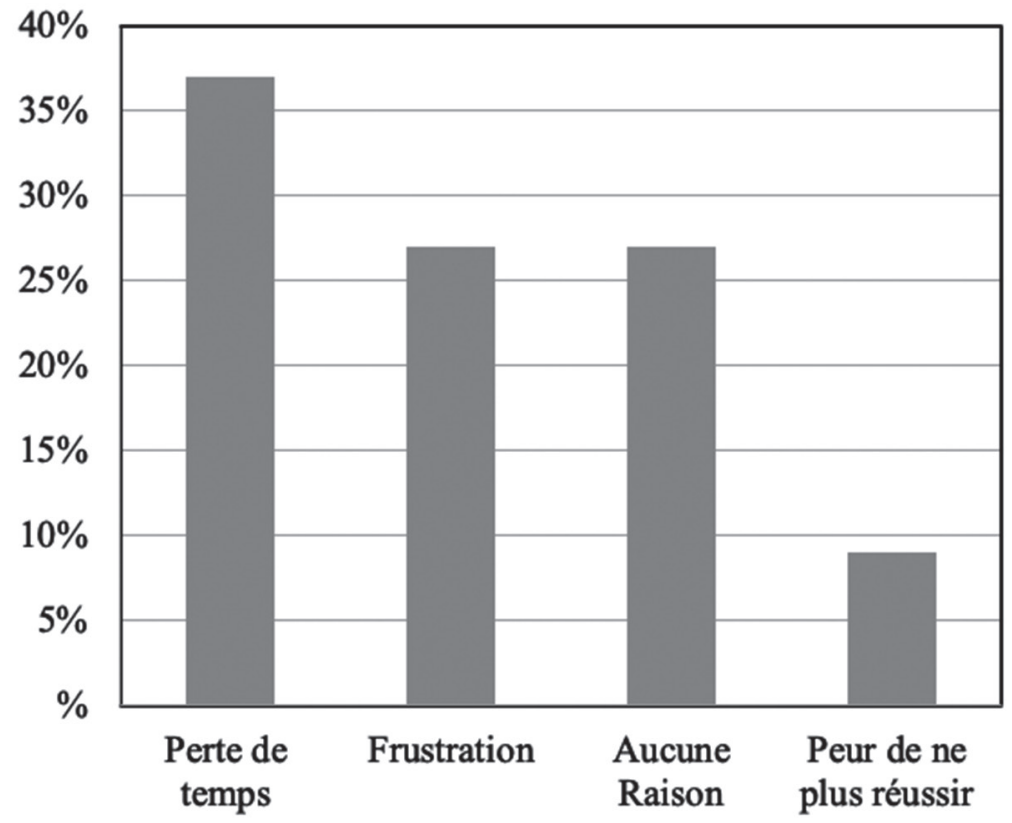

Figure 3. Facteurs négatifs présents après avoir changé de programme. 
Les stratégies du professeur pour surmonter les difficultés face au redoublement

Les étudiants estiment que la stratégie la plus efficace pour surmonter les difficultés liées au redoublement consiste à utiliser une méthodologie capable de favoriser l'acquisition des connaissances, afin de clarifier les doutes sur les sujets et de motiver les étudiants à poursuivre leurs études. En plus, les participants considèrent que les enseignants devraient utiliser peu de méthodes traditionnelles en classe et donner plus de cours audiovisuels ou inversés.

En ce qui concerne le développement du cours, les stratégies d'enseignement les plus employées par les professeurs afin de surmonter les difficultés face au redoublement - sont les suivantes: des activités avec des textes à compléter, des exposés, des fiches de soutien, des activités en classe, des livres avec audio, des guides à remplir et des tutorats.

De plus, l'enseignant utilise aussi des chansons pour compléter les paroles et des vidéos sur Internet pour améliorer la prononciation et l'écoute, en faisant des exercices ou des exemples en classe. Si quelqu'un ne comprend pas, il explique de manière personnelle, dans un tutorat ou avec du matériel de soutien, et même, il fait des jeux de rôles en mettant en œuvre la thématique travaillée. De même, s'il est nécessaire, il utilise la méthode de français "Écho " ou, si le sujet est très long, il emploie le tableau de manière claire et ordonnée pour mieux se faire comprendre, puis les étudiants font des exercices et des productions orales ou écrites.

Parfois, l'enseignant explique le sujet à l'aide de diaporamas et il évalue la thématique par écrit et oralement.
En outre, il utilise des liens sur Internet pour pratiquer le nouveau vocabulaire. Cependant, quand il donne aux étudiants des guides qui sont parfois très longs ou il laisse des exercices pour travailler à la maison, ceci les décourage. Il fait aussi différentes activités, mais elles sont toujours très courtes. Pendant les cours, les enseignants développent de nouveaux thèmes, cela devient monotone et parfois ennuyant, car les sujets ne sont pas très clairs. Par contre, selon les étudiants, l'enseignant doit chercher des pages alternatives pour pouvoir engager une conversation avec de personnes natives.

\section{Les stratégies utilisées en FLE afin d'éviter l'échec et le décrochage}

Certains étudiants affirment que les enseignants n'utilisent pas de stratégies spécifiques pour le déroulement d'un cours. Cependant, afin de renforcer les thématiques travaillées et de faire une rétroaction pendant le processus d'apprentissage d'une langue étrangère, ils doivent faire des débats ou des activités grâce auxquelles les apprenants parlent d'un sujet d'intérêt et d'actualité. C'est pourquoi les activités utilisées par eux pour améliorer leur processus d'apprentissage et éviter ainsi de redoubler un cours sont les suivantes: pour la compréhension écrite, chercher des textes afin d'améliorer l'écriture, d'acquérir un nouveau vocabulaire et ainsi mieux comprendre le sujet sur lequel ils travaillent; faire du travail en groupe; travailler de manière autonome, comme lire, faire des fiches contenant les conjugaisons des verbes, faire des exercices en ligne pour corriger les erreurs, suivre des cours en ligne, regarder des vidéos sur Internet, 
écouter de la musique dans une autre langue pour apprendre à prononcer correctement, compléter des chansons pour améliorer la compréhension orale, en enrichissant le vocabulaire et en améliorant la prononciation, étudier avec une autre personne et utiliser des applications pour parler avec des amis étrangers. En plus, les étudiants mentionnent que les tutorats réalisés par l'enseignant les aident également à mieux comprendre les thématiques du cours, dans lesquelles ils ont des lacunes. Comme les espaces de tutorats sont personnalisés et très efficaces, c'est alors le moment où ils demandent à l'enseignant de clarifier leurs doutes car plusieurs fois en classe, les professeurs ont déjà mesuré leur temps et de réaliser ce qu'ils avaient déjà planifié.

\section{Perceptions des apprenants de FLE niveau B1 par rapport au redoublement}

Dans l'analyse des données, les chercheurs constatent que le redoublement d'un cours provoque des conséquences positives et négatives chez les étudiants. Dans ce cas, le participant 4 affirme que " répéter un cours a ses points forts et ses points faibles. D'une part, parce que nous pouvons renforcer le sujet dans lequel nous avions des lacunes ou que nous n'avions pas bien compris la première fois que nous l'avons vu. D'autre part, nous restons dans le même cours ou nous ne sommes pas à l'aise avec les camarades de classe".

Premièrement, le redoublement d'un cours provoque des points forts qui se sont reflétés dans la vie académique des participants redoublants: $43 \%$ motivateur, $29 \%$ responsabilité, $21 \%$ persistant et $7 \%$ auto-efficacité (Figure 4). Les étudiants se sentent motivés à continuer le cours car ils se sentent plus rassurés par rapport à la méthodologie employée par l'enseignant. Ils comprennent aussi les sujets et apprennent le nouveau vocabulaire tout en mieux connaissant leurs nouveaux camarades.

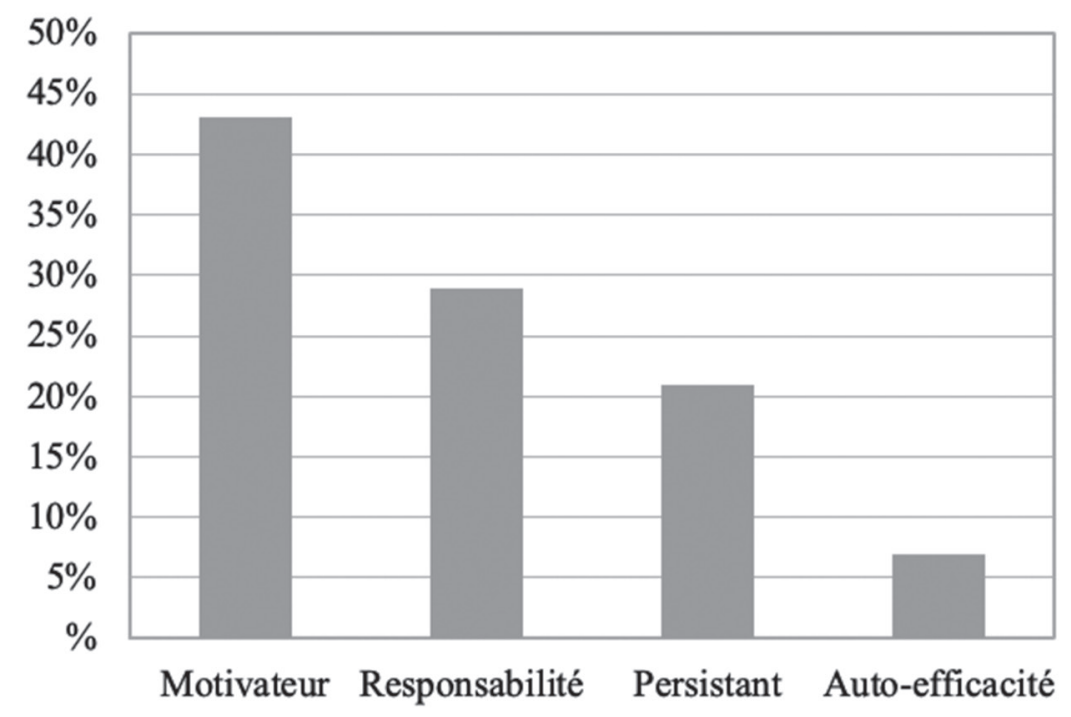

Figure 4. Facteurs positifs reflétés dans la vie académique des redoublants. 
Cependant d'autres facteurs programme, les résultats sont très - plutôt positifs- ont été reflétés dans encourageants. Par exemple: 39\% mola vie académique des étudiants dé- tivateur, $22 \%$ responsabilité et persiscrocheurs après avoir changé de tant, $17 \%$ auto-efficacité (Figure 4).

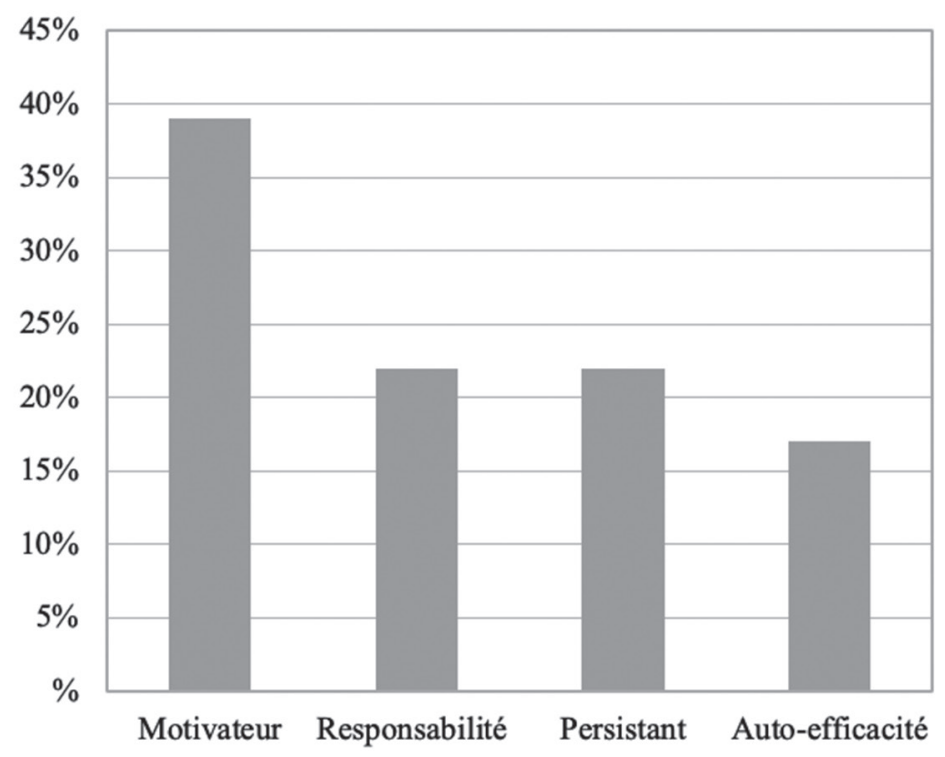

Figure 5. Facteurs positifs reflétés dans la vie académique des décrocheurs.

En deuxième lieu, les étudiants affirment que les conséquences négatives présentes dans leur vie académique sont dues à la perte de temps et d'argent, le jugement de la part de leur famille et leurs camarades, le manque de sécurité et de confiance en soi même, la déception, la frustration, le stress, la perte d'amitiés et d'opportunités entre autres.

Enfin, le redoublement possède une contrepartie car il est aussi essentiel afin de renforcer les aspects à corriger ce qui aide les nouveaux camarades de classe à bénéficier des connaissances sur le sujet, ainsi il permet d'obtenir des avantages favorables, tels qu'apprendre des erreurs pour améliorer chaque jour, le renforcement des échecs, l'éclaircissement des doutes et l'acquisition de nouvelles connaissances indispensables pour compléter les objectifs proposés dans le plan d'études de la licence.

D'après l'expérience du participant 3 « le redoublement génère plus de connaissances, aide à clarifier les doutes et à perdre la peur au moment de parler. De plus, les mêmes compagnons vous demandent de les aider quand ils ne comprennent pas un sujet, donc vous pouvez l'expliquer à nouveau, car vous avez une connaissance préalable». Par conséquent il est évident que le redoublement provoque chez les étudiants de la licence un manque d'intérêt. Cela entraine à son tour de l'inattention et une faible performance, comme ne pas comprendre les aspects fondamentaux de l'écriture et de la prononciation d'une deuxième langue qui se traduisissent en un esprit 
d'irresponsabilité de l'étudiant dans le cours. Selon les expériences du participant 2 et 1 , il est possible de constater que le redoublement est provoqué par des raisons provenant des participants. Elles expliquent que " pour ne pas étudier pour mes quiz, pour avoir des notes faibles dans mes travaux scolaires et pour le manque d'attention et d'engagement de ma part " et encore " peut-être que ma méthode d'étude n'est pas adéquate. En classe, je suis parfois très déconcentrée avec d'autres choses. Chez moi, je suis très peu autonome, je ne trouve pas de méthode adéquate pour étudier et pour que mon apprentissage soit meilleur $"$.

\section{Facteurs causant l'échec et le décro- chage d'un cours}

Selon les expériences personnelles des étudiants redoublants, les facteurs provoquant l'échec d'un cours sont liés au manque d'engagement (35\%), au manque de responsabilité (30\%), aux notes $(25 \%)$ et au manque de connaissances et de travail (5\%). Ils sont aussi liés aux méthodes d'étude et à l'apprentissage, et même à la faible performance académique (35\%), à la méthodologie du cours (20\%) et aux problèmes personnels (10\%). Cependant, l'échec n'a pas été associé à la qualité du programme, aux difficultés économiques et à l'entourage universitaire (Figure 6).

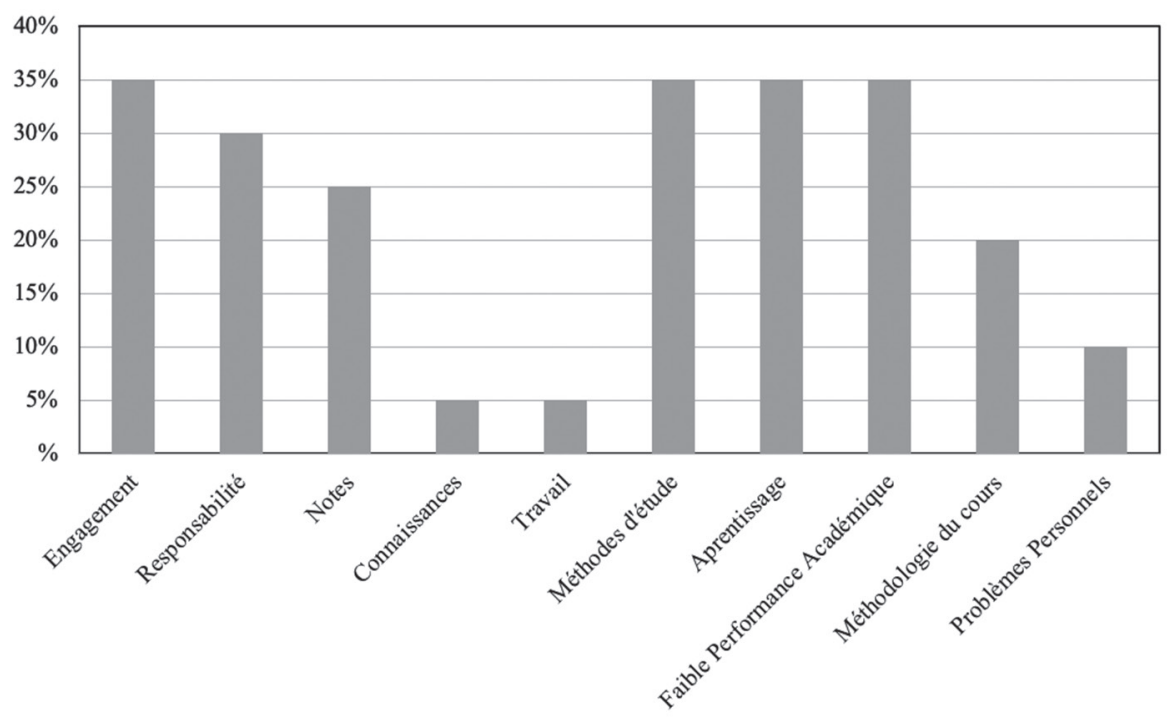

Figure 6. Facteurs provoquant l'échec d'un cours.

Les participants redoublants mentionnent les aspects influençant le décrochage d'un cours, $28 \%$ affirme que leur décision dépend de la faible performance académique (méthodes d'étude), 24\% méthodologie d'enseignement et à l'évaluation des professeurs, $12 \%$ motivations avec le programmeoul'université, aux horaires des cours et aussi à la responsabilité et à l'intérêt de l'étudiant pour apprendre et à des problèmes d'ordre personnel. Cependant, le décrochage n'a pas été lié aux difficultés économiques et à la relation compagnons-professeurs (Figure 7). 


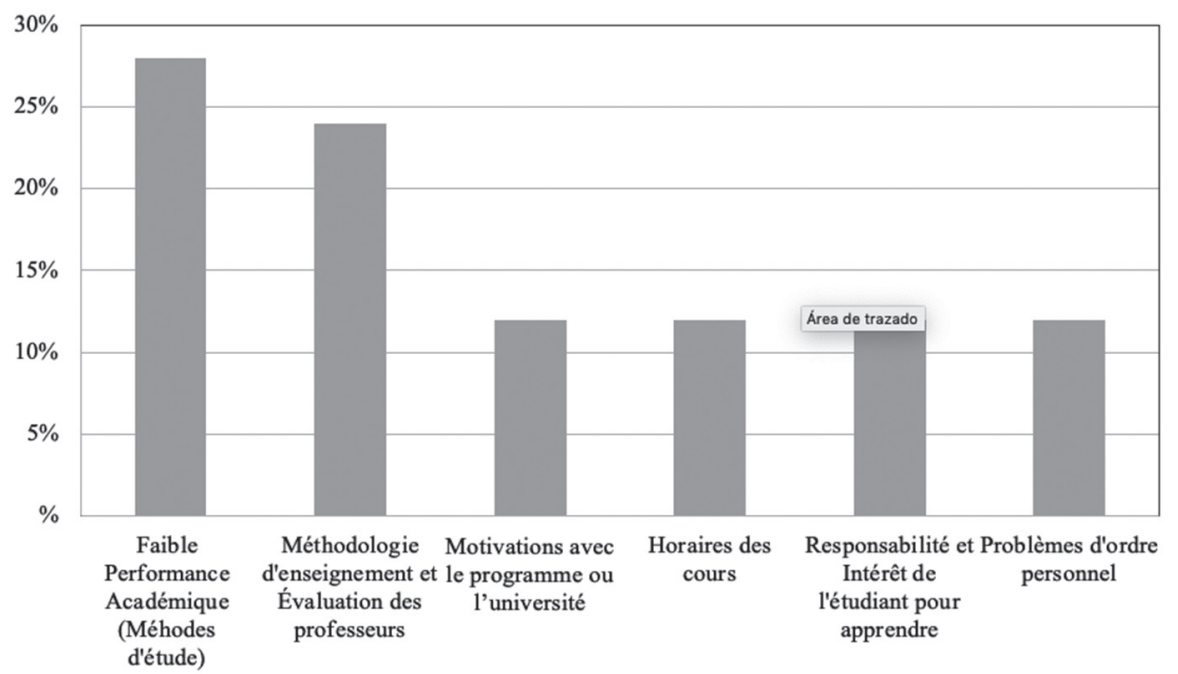

Figure 7. Aspects influençant le décrochage d'un cours.

En outre, les participants exposent personnels et l'interaction profesqu'ils ont abandonné le cours pour seur-étudiant et $8 \%$ faible performance $39 \%$ méthodologie du professeur, 23\% académique (Figure 8). entourage du cours, $15 \%$ problèmes

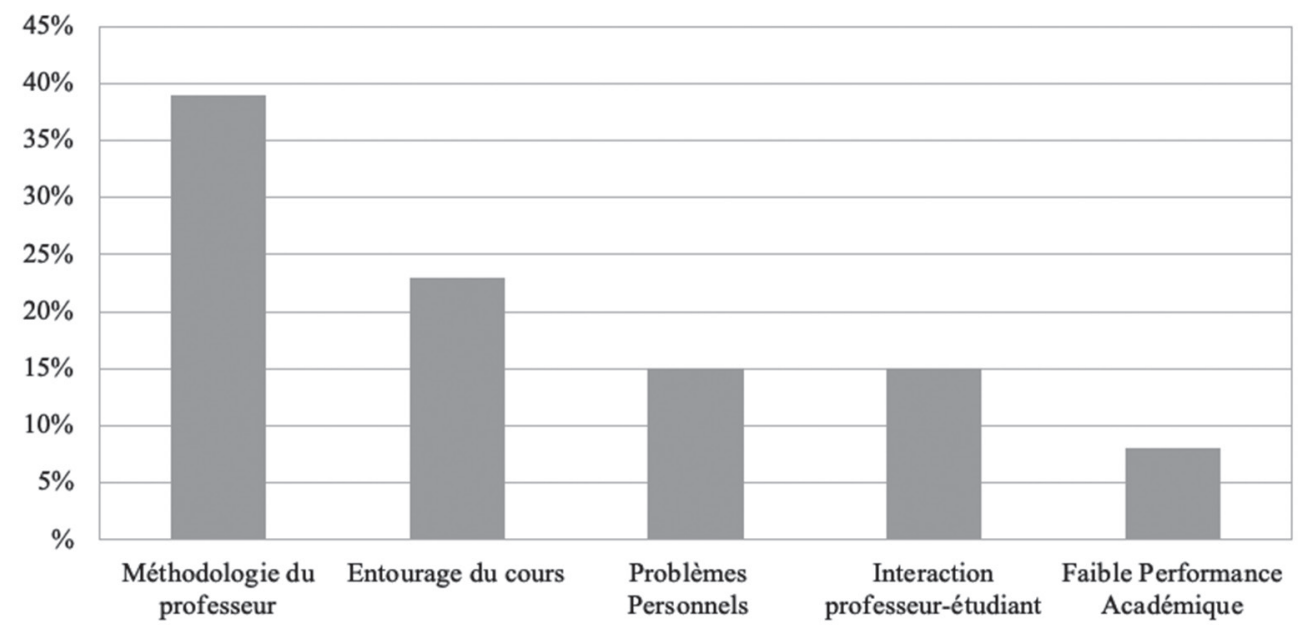

Figure 8. Autres aspects influençant l'abandon d'un cours. 
Les étudiants indiquent aussi les 14\% estiment que l'abandon est lié à la impacts d'avoir raté ou abandonné un perte de confiance (estime de soi), 9\% cours: $23 \%$ affirment qu'il y eu une amé- au sentiment d'infériorité et juste et lioration de la thématique (défauts) $4 \%$ à la peur de ne plus être avec des et une démotivation, 18\% indiquent collègues (Figure 9).

une acquisition de connaissances,

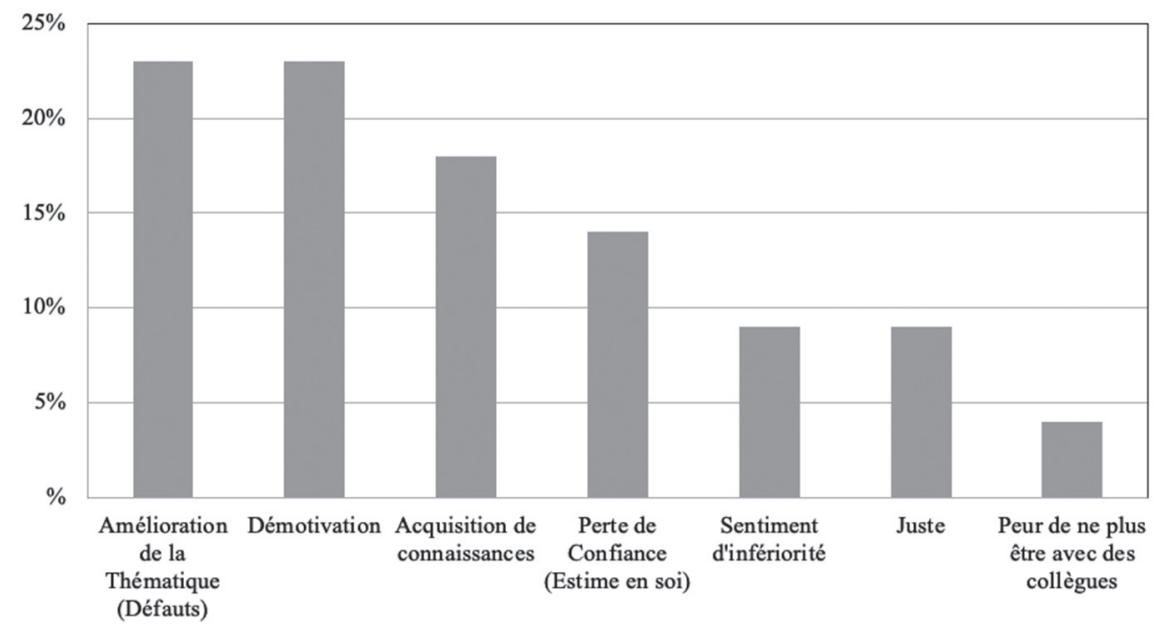

Figure 9. Impacts d'avoir raté ou abandonné un cours.

C'est pourquoi, l'échec et le décrochage $27 \%$ psychologique, $20 \%$ sociologique et génèrent certains changements au ni- 13\% personnel (Figure 10). veau: $40 \%$ académique - organisationnel,

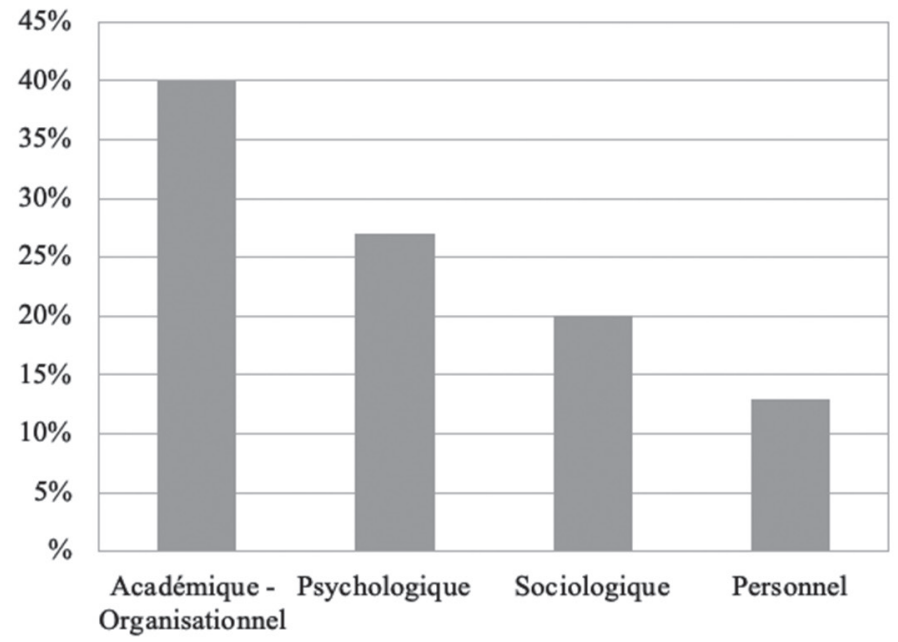

Figure 10. L'échec et le décrochage d'un cours génèrent certains changements au niveau. 
Premièrement, les participants énoncent que le redoublement d'un cours peut amener leurs parents à perdre l'intérêt de les aider et ainsi ils ne peuvent pas continuer leurs études. Le participant 5 déclare que « dans mon cas, cela n'a pas eu lieu, mais les parents peuvent vous dire, je ne vous aiderai plus et peux vous retirer l'aide économique. Cela dépend du redoublant s'il décide de continuer seul ou s'il laisse le programme; je pense que c'est un obstacle, le plus difficile à surmonter ", tandis que selon le participant 6 « les parents seront déçus par la perte de travail et d'argent quand leurs enfants répètent, ils pourront leur reprocher et que cela aura un impact psychologique ». Les étudiants mentionnent aussi que le manque d'engagement, d'intérêt, d'attention et de responsabilité les affecte car ils sont incapables de travailler de manière autonome afin d'acquérir une méthode d'étude idéale pour apprendre une seconde langue.

En deuxième lieu, la performance académique a été aussi affectée due aux faibles bases académiques, aux notes et à la méthodologie développée par les professeurs pendant l'enseignement du français. Le participant 3 mentionne que " j'ai dû annuler le cours en raison d'une mauvaise performance » ; le participant 1 « la dernière fois que j'ai décidé de quitter le cours, c'était parce que j'avais la moyenne très basse et que je ne me sentais pas capable de surmonter cette note. De la même manière, le professeur était très exigeant avec les travaux scolaires, j'ai senti une dépression alors j'ai décidé de le laisser » et le participant 4 " quand je sais que je dois suivre des cours avec le même enseignant du semestre précédent et que je n'ai pas aimé sa méthodologie, je change de groupe $"$.

En troisième lieu, les participants expriment que la méthodologie de l'enseignant est essentielle pour être motivé à continuer la licence, car il est important que les enseignants donnent des cours dynamiques qui s'adaptent aux styles d'apprentissage des étudiants. Dans le cas du participant 6 «la raison principale était la méthodologie employée par l'enseignant, qui ne s'exprimait pas correctement, il n'avait pas vraiment une méthode de travail intéressante, il y avait souvent des guides d'exercices à faire et qui résumaient trois sujets dans un cours. Il n'y avait pas de vrai approfondissement du sujet et quand il le faisait, c'était une explication minimale qui semblait très obsolète. Je n'ai pas aimé la façon de faire les cours, il n'a pas de charisme, il n'a pas de capacité linguistique pour s'exprimer et motiver la classe».

\section{Facteurs causant l'échec et le décro- chage d'un programme}

Selon les expériences personnelles d'étudiants décrocheurs, les facteurs provoquant l'échec d'un programme sont liés à des aspects tels que: les notes (40\%), aucune raison (20\%), au manque d'engagement, au manque de connaissances et de responsabilité, et un autre choix: la frustration (10\%). Cependant, il n'a pas été lié au manque de travail (Figure 11). 


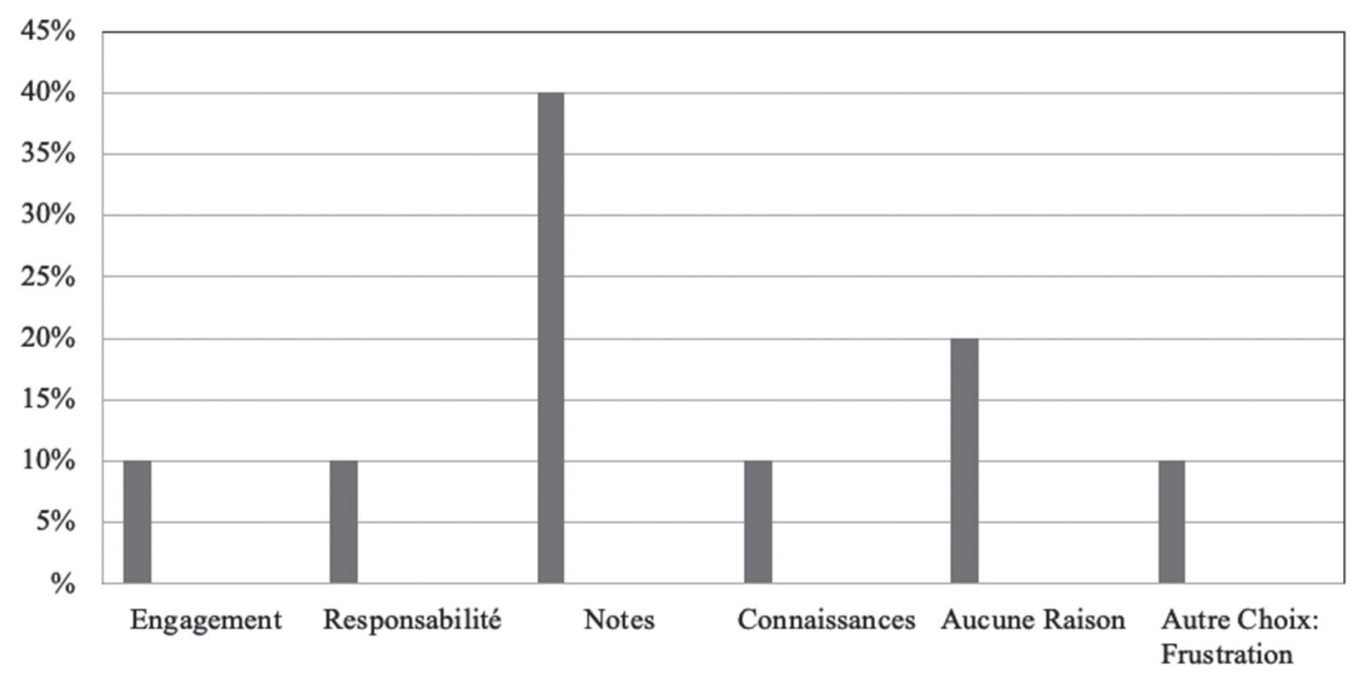

Figure 11. Facteurs provoquant l'échec d'un programme.

Les participants décrocheurs mentionnent les aspects influençant l'abandon d'un programme: $27 \%$ affirme que leur décision dépend des méthodes d'enseignement et de l'évaluation de la part des professeurs, $23 \%$ des relations avec leurs compagnons et professeurs, $19 \%$ des motivations avec le programme ou l'université, $15 \%$ lié à la faible performance académique (méthodes d'étude), $12 \%$ aux problèmes personnels (famille, maladies...), 4\% aux difficultés économiques. Cependant, le décrochage n'a pas été lié aux horaires des cours, et à la responsabilité ou à l'intérêt de l'étudiant pour apprendre non plus (Figure 12).

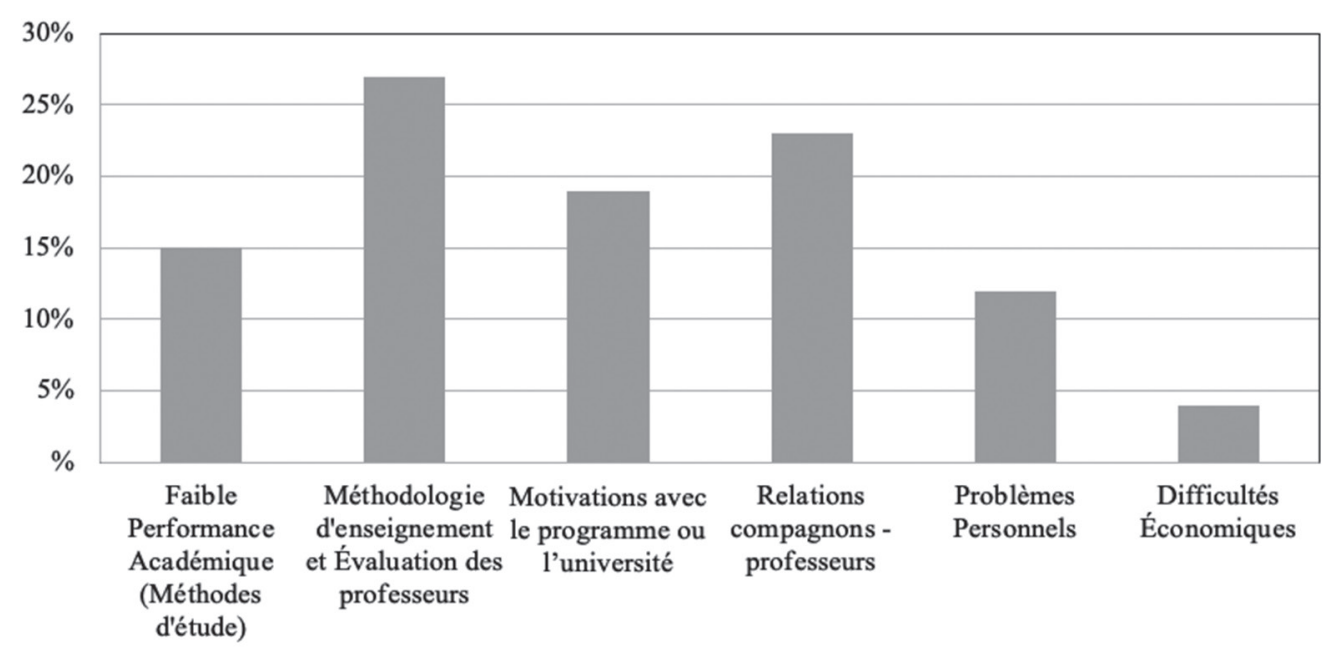

Figure 12. Aspects influençant l'abandon d'un programme. 
Les étudiants indiquent aussi les impacts d'avoir changé du programme: $21 \%$ affirment qu'il y eu une amélioration de la thématique (défauts), 16\% indiquent une acquisition de connaissances, la démotivation, aucun im- pact, $11 \%$ disent que c'est une seconde chance, $10 \%$ affirment que l'abandon est lié à la peur de ne plus être avec des collègues, $5 \%$ estiment que c'est lié à la perte de confiance (estime de soi) et sentiment d'infériorité (Figure 13).

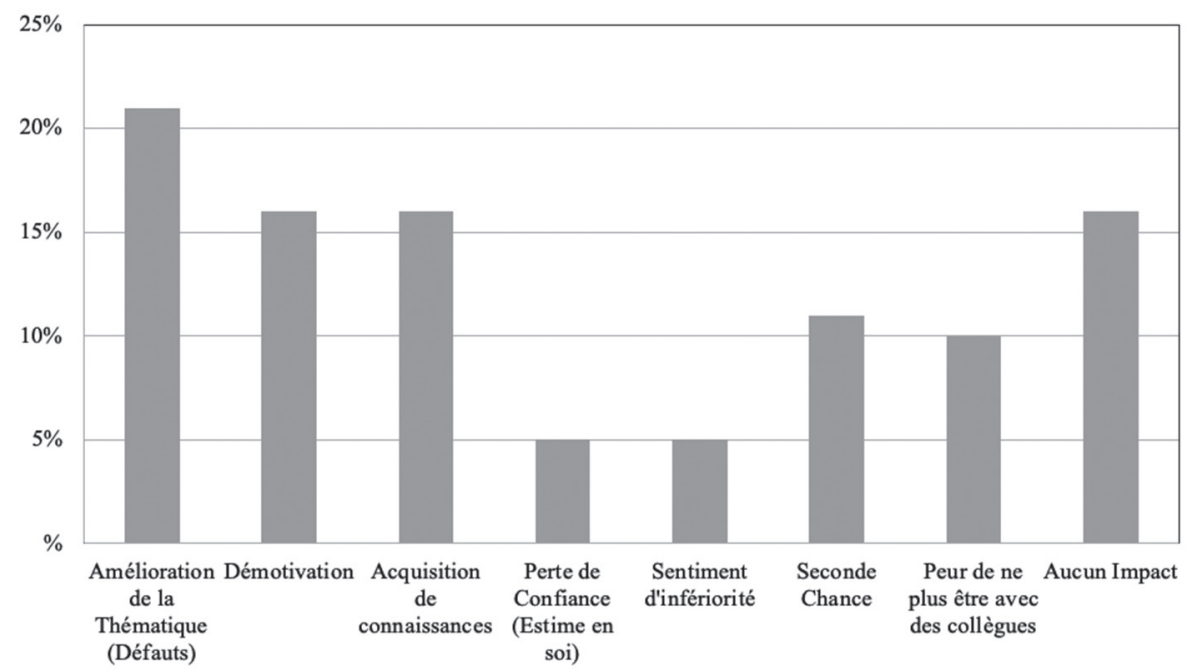

Figure 13. Impacts d'avoir changé du programme.

C'est pourquoi, l'échec et le dé- $17 \%$ psychologique, $8 \%$ personnel, inscrochage génèrent certains change- titutionnel et économique (Figure 14) ments au niveau: 59\% académique,

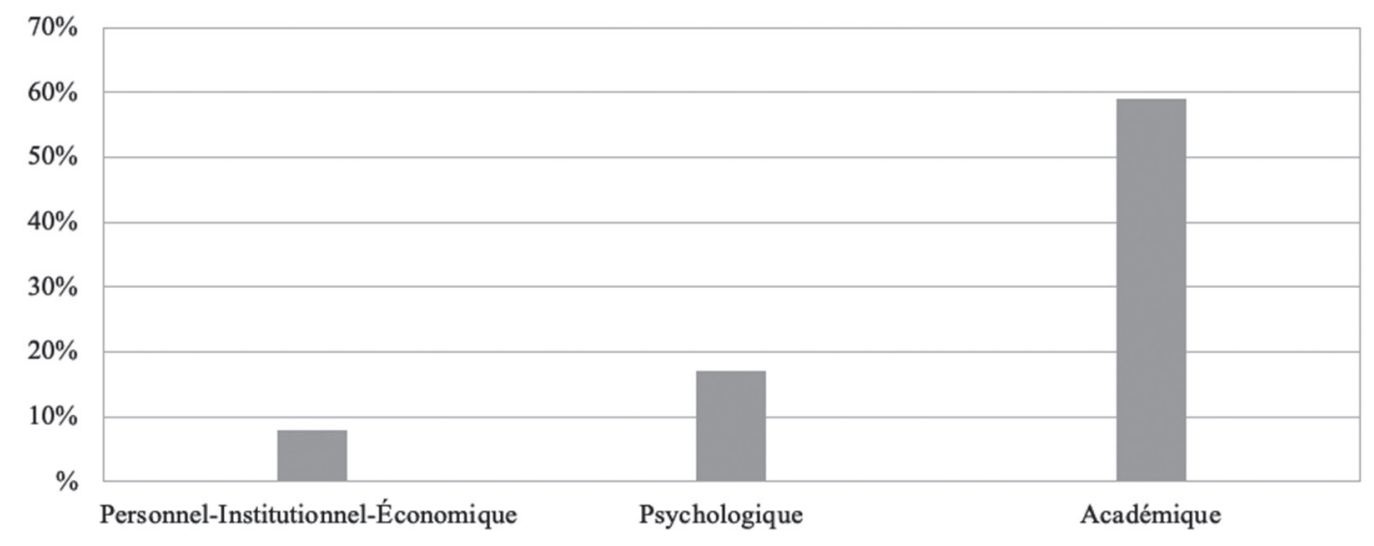

Figure 14. L'échec et le décrochage d’un programme génèrent certains changements au niveau. 
D'ailleurs, tenant compte les expériences des participants décrocheurs, le changement de programme était le meilleur choix, car ils ont appris des thématiques variées et ont amélioré leurs notes. Cependant, certains d'entre eux considèrent qu'ils ont pris la décision trop tard ou en raison de facteurs externes. Par exemple, le participant 7 énonce que " ma mauvaise décision était de ne pas avoir changé avant à la licence en espagnol où je suis satisfaite", tandis que le participant 8 affirme que " je considère que c'était une décision erronée, j’ai pris cette décision en raison des circonstances économiques qui ont influencé ma performance académique ». En plus, la Licence en Langue espagnole et communication était la deuxième option pour certains étudiants, ce qui favorise un apprentissage plus approfondi de la langue maternelle et a facilité l'homologation des sujets. Selon le cas du participant 9 « pour apprendre une autre langue, j'ai d'abord dû améliorer ou parfaire ma langue maternelle, j'ai amélioré mon lexique, mon orthographe, ma grammaire et ma compréhension écrite».

\section{Discussion}

Les résultats de cette étude indiquent que le redoublement et le décrochage d'un cours sont des phénomènes présents dans la vie universitaire des étudiants, provoquant des conséquences positives dans leur vie académique car il permet aux étudiants de surmonter leurs faiblesses, éclaircir leurs doutes et acquérir de nouvelles connaissances requises afin d'accomplir les objectifs proposés dans le plan d'études des licences.
En outre, le redoublement cause des conséquences négatives, car il génère des changements au niveau émotionnel et psychologique. Les étudiants ressentent une série d'émotions telles que la frustration, la déception, le désintérêt, et aussi tous les aspects émotionnels liés à la perte et à l'échec. Ce qui cause une altération dans la confiance et la motivation des étudiants pour continuer les études. Au niveau académique, ceci cause des défauts dans l'acquisition des connaissances et dans le processus d'apprentissage d'une langue étrangère. De même, les étudiants estiment que la stratégie la plus efficace pour surmonter les difficultés liées au redoublement consiste à utiliser, de la part du professeur, une méthodologie capable de les motiver à poursuivre leurs études car il est important que les enseignants réalisent des cours dynamiques et qui s'adaptent à leurs styles d'apprentissage, où ils développent des exercices, des textes à compléter, des exposés, des fiches de soutien, des guides à remplir et des tutorats afin de clarifier leurs doutes par rapport aux thématiques travaillées et de faire une rétroaction pendant leurs processus d'apprentissage.

En ce qui concerne le décrochage d'un cours, les étudiants expriment que leur décision d'abandonner les études dépend de la faible performance académique (méthodes d'étude), de la méthodologie d'enseignement et l'évaluation des professeurs, de la responsabilité et de l'intérêt pour apprendre et des problèmes personnels. Les apprenants mentionnent aussi que le manque d'engagement, d'intérêt, d'attention et de responsabilité les affectent car certains d'entre eux sont incapables de travailler de manière autonome et 
acquérir une méthode d'étude idéale pour apprendre une langue étrangère.

Pendant le déroulement de cette étude, les chercheurs ont eu une limite liée au temps. Tenant compte que les étudiants n'avaient pas le même calendrier académique que le leur, il n'a pas été possible d'établir une heure précise pour le développement des groupes focalisés donc ils ont été déroulés avec un nombre limité des participants. Cependant, cette limite n'a pas empêché de collecter les données nécessaires liées au phénomène d'étude.

Ce projet sert à la communauté éducative de la licence de langues étrangères et à l'université de Pamplona pour la mise en œuvre d'un plan d'amélioration afin de réduire le taux d'abandon universitaire, offrant une vision de l'importance des langues étrangères dans la vie professionnelle des étudiants. De plus, cette étude sert aussi à aborder une future recherche liée au décrochage tenant compte le dispositif de " Alertas Tempranas " développé par le " Bienestar Universtario " comme un outil de soutien académique afin d'éviter le redoublement et le décrochage chez les étudiants et donc établir son rôle dans le processus d'apprentissage d'une langue étrangère.

\section{Conclusion}

Les perceptions des apprenants face au redoublement au cours de la scolarité obligatoire indiquent que ce phénomène est perçu dès deux points de vue. D’une part, il existe la comparaison entre la pensée des personnes face à la pratique du redoublement comme un fait utile et nécessaire au niveau éducatif. D'autre part, il y a une analyse de sa réalité inefficace concernant le progrès cognitif, la motivation et la méthodologie des enseignants. Cette étude a offert un regard de certains facteurs internes et externes, afin de présenter le panorama de ce phénomène (redoublement) à l'Université de Pamplona. L'étudiant a recours à abandonner un programme, en raison de ses faibles compétences au niveau académique, de sa démotivation, de son insatisfaction et de l'humiliation par rapport aux autres camarades. En plus, le redoublement d'un cours avec le même enseignant, le retard d'un semestre, la perte de temps et d'argent et la méthodologie d'enseignement utilisée par certains professeurs de la Licence en Langues étrangères amènent les étudiants à changer de programme, influençant négativement leur processus d'apprentissage. De plus, les participants considèrent que la méthodologie, l'accompagnement et les contenus programmatiques enseignés par les professeurs ne sont pas adéquats, puisqu'ils ne cherchent pas de stratégies pour combler et anéantir les défauts de la langue chez les apprenants. Les stratégies mises en œuvre doivent être conçues en fonction des étudiants. Par contre, les difficultés avec la langue française provoquent des défauts dans l'acquisition des connaissances et cela devient donc un processus inefficace d'apprentissage d'une langue étrangère. 


\section{Bibliographie}

Beaud, S. (2005). L'échec à l'université des " enfants de la démocratisation " Une question occultée. Repéré sur https://archive.boullier.bzh/cosmopolitiques_com/cosmopolitiques_com_ archive_boullier_bzh_Beaud\%20 L'\%C 3\%A9chec\%20\%C 3\%A0\%20 l'universit\%C3\%A9\%20des\%20enfants $\% 20 \mathrm{de} \% 201 \mathrm{la} \% 20 \mathrm{~d} \% \mathrm{C} 3 \% \mathrm{~A} 9 \mathrm{mo}-$ cratisation.pdf

Beaupère N., Boudesseul G. et Macaire S. (2009) in Sarfati , F. (2015). "L'université face au décrochage". La Vie des idées - ISSN : 2105-3030. Chauveau, G. et Rogoas-Chauveau, E. (1996) Chargés de recherche CRESAS-INRP L'échec scolaire existet-il ?, in Echec et réussite scolaires, Revue Migrants-formation, $\mathrm{n}^{\circ} 104$, p.12 Retrouvez plus de ressources sur www.citoyendedemain.net

Chenu, F. et Blondin, Ch. (n.d). Décrochage et abandon scolaire précoce.

Conseil de l'Union européenne (2011). Recommandation du Conseil du 28 juin 2011 concernant les politiques de réduction de l'abandon scolaire. Journal officiel de l'Union européenne C 191. Repéré sur http://eur-lex.europa.eu/ LexUriServ/LexUriServ.do?uri=OJ:C:2011:191:0001:0006:fr:PDF

Conseil National d'Évaluation du Système Scolaire - CNESCO (2016). Inégalités sociales et migratoires : comment l'école amplifie-t-elle les inégalités?

Cosnefroy, O. et Rocher, T. (2005). Le Redoublement au cours de la scolarité obligatoire : nouvelles analyses, mêmes constats.

Crahay (1996, 2000) ; Paul (1996) in Cosnefroy, O. et Rocher,T. (2005).
Le redoublement au cours de la scolarité obligatoire : nouvelles analyses, mêmes constats.

Creswell, J.W. (1998). Qualitative inquiry and research design. Choosing among five traditions. Thousand Oaks, CA : Sage.

Creswell, J.W. (2012). Educational Research: Planning, Conducting, and Evalualing Quantitative and Qualitative Research.

Creswell, J. W. et Plano Clark, V.L. (2006). Designing and Conducting Mixed Methods - Research. 1er éd. Sage Publications, Inc.

Draelants, H. (2006). Le redoublement est moins un problème qu'une solution. Comprendre l'attachement social au redoublement en Belgique francophone. halshs-00563874.

Fonds International des Nations Unies pour l'Enfance - UNICEF (2015). Analyse de la Situation des enfants dans le monde: Réimaginer l'avenir - Colombie. Repéré sur https:// www.unicef.org/french/infobycountry/colombia_2660.html

Giorgi, A. (1997) in St-Germain, D., Delpêche, L et Mercier, D. (2009). L'informatique comme soutien à l'opérationnalisation des procédures analytiques en phénoménologie : un modèle de développement et de collaboration. Recherches Qualitatives - Vol. 28(1), 106-132. ISSN 1715-8702.

Hababou (n.d). Les méthodes d'échantillonnage. Repéré sur https:// d1n7iqsz6ob2ad.cloudfront.net/.../ pdf/53340ca9cc969.pdf

Johnson, R.B. et Onwuegbuzie, A.J. (2004). "Mixed Methods Research: A Research Paradigm Whose Time Has Come." Educational Researcher 33:14-26. 
Ketele, J. -M. et Roegiers, X. (2015). Méthodologie du recueil d'informations. Fondements des méthodes d'observation, de questionnaire, d'interview et d'étude de documents (5e éd.).

Kitzinberg, J., Marková, I., et Kalampalikis, N. (2004). Qu'est-ce que les focus groups? Bulletin de psychologie, 57(3), 237-243.

Lafontaine, D. et Crahay, M. (2004). Échec et décrochage scolaires en Communauté française de Belgique, Revue internationale d'éducation Sèvres, 35, 55-66. Repéré sur http:// orbi.ulg.ac.be/handle/2268/8010

Lozano, M. (2013). A phenomenological study on FL learner's dropout at a public university. Université de Pamplona, Colombie. Document de travail.

Ministerio de Educación Nacional de Colombia (2015). Estrategias para la permanencia en educación Superior: experiencias significativas.

Monseur, C. et Crahay, M. (2008). Composition académique et sociale des établissements, efficacité et inégalités scolaires : une comparaison internationale, Revue Française de Pédagogie, 164, 55-65.

Paul, J.-J. (n.d). Dans quels cas peuton refuser / faire appel d'un redoublement? Repéré sur https://www. magicmaman.com/,dans-quelscas-peut-on-refuser-le-redoublement,50,724.asp

Paul, J.-J. (1996). Le redoublement : pour ou contre? Paris : ESF éditeur.

Sarfati, F. (2015). "L'université face au décrochage ». La Vie des idées ISSN : 2105-3030.

Statistique Canada (2003). Méthodes et pratiques d'enquête. Repéré sur http://www.caricomstats.org/helpdesk/documentarycentre/Dom4/ StatCan_M\%C3\%A9thodesPrat iquesEnqu\%C3\%AAtes_12-587x2003001-fra.pdf

Tejada, Ch. (2015). L'impact de représentations des élèves sur l'efficacité de leur redoublement en cycle terminal du lycée technologique.

Touboul, P. (n.d). Recherche qualitative : La méthode des Focus Groupes - Guide méthodologique pour les thèses en Médecine Générale. 
